\title{
Capsule Commentary on Baig et al., Picture Good Health: A Church-Based Self-Management Intervention among Latino Adults with Diabetes
}

\author{
Jeff Whittle, MD MPH \\ Clement J. Zablocki VA Medical Center, Milwaukee, WI, USA.
}

J Gen Intern Med 30(10): 1534

DOI: $10.1007 / \mathrm{s} 11606-015-3375-6$

(c) Society of General Internal Medicine 2015

$\mathrm{T}$ here is strong and growing interest in the potential for community members to help one another in coping with chronic disease. Community members with either personal or shared experience with a chronic disease likely have a better sense than most healthcare professionals regarding feasible disease self-management activities for affected persons in their community. Moreover, they are more apt to communicate in a style with which the learner is more familiar; learners working with a peer may be more willing to risk asking questions that could make them appear uneducated. This improved bidirectional communication may help persons with chronic disease to better understand and implement effective self-management behaviors. There is considerable empiric support for this belief, particularly for diabetes. ${ }^{1}$

On the other hand, we should not undervalue the contribution of healthcare professionals' more complete and nuanced understanding of disease pathophysiology, the variability of presentation and prognosis, and the range of treatment options. Studies of self-management support for hypertension in particular tend to show that including a healthcare professional who can adjust medications (or cause them to be adjusted) is an important driver of impact. ${ }^{2}$

Particularly for diabetes, where both lifestyle and medication have major contributions, the need for a collaborative approach seems clear. However, it is not clear how to operationalize this partnership. ${ }^{3}$ While the present intervention led to, at best, modest changes in self-reported health behaviors, Baig and colleagues have developed a relationship with their community partners. ${ }^{4}$ This working relationship may well allow them to overcome the social and logistic challenges of sharing resources and workload between healthcare professionals and community members, groups that may share long-term goals but have very different ideas regarding methods and research approaches. ${ }^{5}$ This will be a key challenge as they try to address the idea that future interventions might benefit from stronger linkage to professional healthcare.

Collaboration with established faith communities may be of particular interest, as the religious institution provides community infrastructure that will persist beyond the funding period, as well as a spiritual framework that suggests all members of a community share responsibility for health promotion.

Conflicts of Interest: The author declares that he does not have a conflict of interest.

Corresponding Author: Jeff Whittle, MD MPH; Clement J. Zablocki VA Medical Center, Milwaukee, WI 53295, USA (e-mail: jeffrey.whittle@va.gov).

\section{REFERENCES}

1. Heisler M. Different models to mobilize peer support to improve diabetes self-management and clinical outcomes: evidence, logistics, evaluation considerations and needs for future research. Fam Prac. 2010;27(Suppl 1):i23-i32.

2. Bosworth HB, Powers BJ, Olsen MK, et al. Home blood pressure management and improved blood pressure control: results from a randomized controlled trial. Arch. Intern. Med. 2011;171(13):11731180 .

3. Whittle J. When does peer support improve glycemic control in persons with diabetes mellitus? JAMA Intern Med. 2014;174(6):982-983.

4. Baig AA, Benitez A, Locklin CA, Gao Y, Lee SM, Quinn MT, Solomon MC, Sanchez-Johnsen L, Burnet DL, Chin MH. Picture Good Health: A Church-Based Self-Management Intervention among Latino Adults with Diabetes. J Gen Intern Med 2015. doi: 10.1007/s11606-015-3339-x

5. Whittle J, Fletcher KE, Morzinski J, Ertl K, Patterson L, Jensen W, Schapira MM. Ethical challenges in a randomized controlled trial of peer education among veterans' service organizations. J Empir Res Hum Res Ethics. 2010;4:43-51. 